\section{The influence of pre-mRNA splicing on phenotypic modification in Stickler's syndrome and other type II collagenopathies}

\begin{abstract}
Purpose This paper will illustrate how variation in the processing of mutant pre-mRNA can affect the phenotypic outcome of inherited disorders of type II collagen. Methods Type 1 Stickler's syndrome is one of the different phenotypes resulting from mutations in COL2A1 (the type II collagenopathies). It is also the commonest, but often goes undiagnosed due to the variability of phenotypic features, which in some cases may consist of only abnormal vitreous development. Most cases of type 1 Stickler's syndrome are due to premature termination codons in the mRNA, resulting in haploinsufficiency. This leaves a conundrum as to why the disease is so variable. Using RT-PCR of illegitimate transcript and also minigenes, we have investigated how certain mutations can variably affect mRNA processing. Results Here, we demonstrate and discuss how apparently similar mutations can have a dramatically different effect on splicing of the pre-mRNA, switching transcripts from ones which would be degraded by nonsensemediated decay into messages that will be translated into mutant proteins that can exert a dominant-negative effect and ultimately modify the resulting phenotype.

Conclusion Variability of Stickler's syndrome can, in part, be due to the variable effect that mutations have on the processing of the COL2A1 transcript.

Eye (2008) 22, 1243-1250; doi:10.1038/eye.2008.34; published online 29 February 2008
\end{abstract}

Keywords: Stickler's syndrome; pre-mRNA splicing; phenotypic variability
AJ Richards ${ }^{1}$ and MP Snead ${ }^{2}$

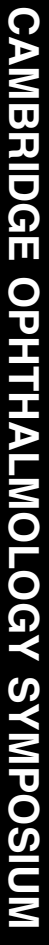

\section{Introduction}

Stickler's syndrome is a genetically heterogeneous disorder affecting the heterotypic (composite) fibrillar collagen molecules found in both vitreous and cartilage. ${ }^{1,2}$ These macromolecules consist of a core of type XI collagen that regulates fibrillogenesis by limiting the addition of the more abundant type II collagen molecules to the collagen fibril. ${ }^{3}$ Situated on the outside of the type II/XI collagen fibril is type IX collagen. This fibril-associated collagen with interrupted triple helices (FACIT) not only connects the collagen fibrils to other components of the extracellular matrix but can also influence spacing between collagen fibrils. ${ }^{4-7}$

Mutations in the genes encoding the constituent proteins affect both vitreous and cartilage tissues. Stickler's syndrome presents with congenital vitreous abnormalities and variable expressivity of other features, such as myopia, premature osteoarthritis, hearing loss, and craniofacial dysmorphology including midline clefting. ${ }^{2}$ The most common form of Stickler's syndrome is type 1 (MIM 108300) and is due to mutations in the COL2A1 gene. ${ }^{8}$ These mutations mostly result in haploinsufficiency of type II collagen due to premature termination of translation, which leads to degradation of the mutant mRNA via the nonsense-mediated decay pathway. ${ }^{9}$ Other types of mutations in this gene, that result in dominant negative effects, can result in much more severe disorders, such as spondyloepiphyseal dysplasia congenita (SEDC) and Kniest dysplasia. ${ }^{10,11}$

Mutations in the genes for type XI collagen result in type 2 (MIM 604841) and type 3 (MIM
'Department of Pathology, University of Cambridge, Cambridge, UK

${ }^{2}$ Vitreoretinal Service, Department of Ophthalmology, Addenbrooke's Hospital Cambridge University Hospitals NHS Trust, Cambridge, UK

Correspondence:

AJ Richards, Department of Pathology, University of Cambridge, Tennis Court Road, Cambridge CB2 1QP, UK. Tel: + 441223 333735;

Fax : +44 1223333730.

E-mail: ar204@

cam.ac.uk

Received: 18 January 2008 Accepted in revised form: 18 January 2008 Published online: 29 February 2008

This work was presented at the XXXVII Cambridge Ophthalmological Symposium 2007. 
184840) Stickler's syndrome. ${ }^{12,13}$ The COL11A1 gene is expressed in both the vitreous and cartilage, mutations here result in the type 2 variety of the disorder, whereas COL11A2 is not expressed in the eye and mutations in this gene result in the 'non-ocular' or type 3 Stickler's syndrome, which only has skeletal and hearing defects. All of these are dominant disorders, however, there has been one report of a recessive form of Stickler's syndrome where patients were homozygous for a premature stop codon in COL9A1. ${ }^{14}$ Interestingly, dominant mutations in type IX collagen genes result in multiple epiphyseal dysplasia (MIM 600204, 600969), but patients with this disorder have not been reported to have any eye pathology. ${ }^{15-17}$

The vitreous phenotype in Stickler's syndrome is the most consistent clinical feature. Type 1 Stickler's syndrome has a typical vestigial vitreous gel in the retrolental space bounded by a folded membrane. ${ }^{2,18}$ In contrast, type 2 Stickler's syndrome is characterised by a vitreous gel filling the posterior segment, but with disorganised gel architecture, including irregularly thickened fibre bundles that have a beaded appearance under slit-lamp examination, which possibly reflects the role of type XI collagen in regulating collagen fibrillogenesis. ${ }^{19}$ Systemic features are variable and although some have an increased frequency in certain types of Stickler's syndrome (ie cleft palate in type 1 and hearing loss in type 2), these differences are not sufficient to accurately predict the mutant locus based on phenotype alone. The variable systemic phenotype extends both between and within families, and as most cases are due to haploinsufficiency of type II collagen, there must be factors other than the mutation that modifies the resulting clinical phenotype.

Genetic association studies have started to identify common DNA variants of genes that result in a predisposition to develop common disorders, such as osteoarthritis (OA) and myopia. ${ }^{20-23}$ It is likely that coinheritance of these genetic predisposition factors is one source of phenotypic variation. For instance, an individual with a mutation causing Stickler's syndrome, who also inherits an OA predisposing gene allele, is more likely to develop this as one of the systemic features than a patient that only has a Stickler mutation and no other predisposing OA alleles. Interestingly, COL2A1, COL11A2, and the type IX collagen genes have been found to be associated with degenerative cartilage disorders and/or myopia. ${ }^{23-25}$ Due to the relatively small numbers of affected individuals with Stickler's syndrome who have been genetically characterised, at present it is not possible to correlate coinheritance of these predisposing risk alleles with the severity of any particular phenotypic trait in this disorder. Instead, our own research has started to examine how apparently similar mutations can have different effects on how the pre-mRNA is processed and how variability in mRNA splicing can potentially affect the resulting phenotype.

\section{Splicing and splicing factors as phenotypic modifiers}

To increase the information carried by the genome, many genes are alternatively spliced, that is, certain exons are either included in or excluded from the mature mRNA transcripts. So similar but subtly different proteins, which can vary in both biochemical characteristics and function, can be synthesised from the same gene. Both COL2A1 and COL11A1 are alternatively spliced. ${ }^{26,27}$ Type II collagen exon 2 is not expressed in mature cartilage but is expressed in the eye. Mutations within this exon are, therefore, naturally removed from the type II collagen transcript in mature cartilage tissue. Patients with these exon 2 mutations typically have a predominantly ocular phenotype with little or no systemic features usually seen in Stickler's syndrome (MIM 609508). ${ }^{28-30}$ In the past, this has resulted in confusion with the rarer Wagner's syndrome (MIM 143200) that lacks systemic features and is due to mutations in the CSPG2 gene that codes for the proteoglycan versican. Although versican is expressed in many tissues, it is also alternatively spliced with four variants that either include or exclude exons 7 and 8 that encode glycosaminoglycan attachment domains. To date, all of the mutations described for Wagner's syndrome affect the splice sites of the alternatively spliced exon $8 .^{31-33}$

Correct pre-mRNA processing requires the accurate recognition of the donor, branch, and acceptor splice sites by the spliceosome, a large RNA/protein macromolecular assembly that removes intronic sequences from the mRNA. To aid the specificity of this process, other transacting splicing factors bind to cis-regulatory sequences in the pre-mRNA. The trans-acting splicing factors can either promote or inhibit formation of the spliceosome at potential splice sites and so the sequences, to which they bind, are known as splicing enhancers or silencers. ${ }^{34}$ Naturally occurring alternative splicing is regulated by controlling the expression of trans-acting splicing factors that can either promote or inhibit exon inclusion in a developmental and cell-type-specific manner.

Alternatively spliced exons often have weak (differ considerably from the consensus sequence) splice sites and are, therefore, more dependant upon trans-acting splicing factors for inclusion into the mature mRNA.

By exploring this potential tissue-specific alternative splicing, we have identified another mechanism resulting in the predominantly ocular variant of Stickler's syndrome. In this case, a mutation altered a donor splice site from Tggtaagc to Tggcaagc. ${ }^{35}$ Donor splice sites with 


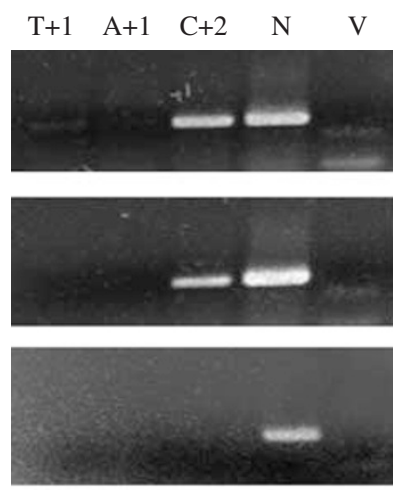

Saos 2

SW1353

OUMS-27

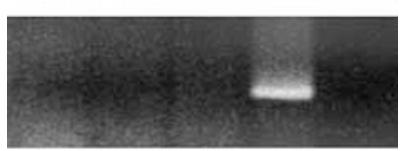

Cornea

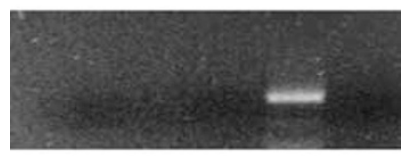

Sclera

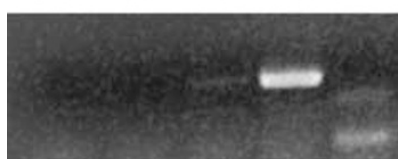

MIO-M1

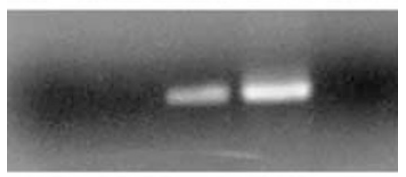

ARPE-19

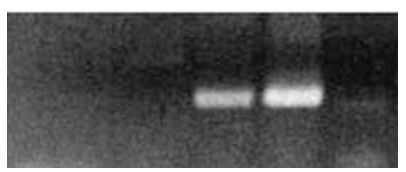

CE11560

Figure 1 A minigene with an IVS51 GC mutant donor splice site can be processed normally. Three different mutations $(\mathrm{G}+1>\mathrm{T}, \mathrm{G}+1>\mathrm{A}$, and $\mathrm{T}+2>\mathrm{C})$ of the intron 51 donor splice site of COL2A1 were cloned as minigenes and transfected into various cultured cell lines as indicated. Minigene-specific RT-PCR using a reverse primer that could only amplify correctly processed transcripts demonstrated that unlike $\mathrm{G}+1>\mathrm{T}$ and $\mathrm{G}+1>\mathrm{A}$, the $\mathrm{T}+2>\mathrm{C}$ mutant was capable of being spliced normally. A minigene with the normal splice site $(\mathrm{N})$ and the cloning vector $(\mathrm{V})$ was used as controls. This mutant resulted in the predominantly ocular phenotype.

gc as the first two nucleotides of the intron occur rarely, but naturally in the human genome and are often alternatively spliced. We demonstrated with the use of minigenes that this gc mutant donor splice site could be spliced normally when transfected into cultured cell lines (Figure 1). However, not all cells were capable of splicing the mutant normally, which presumably was due to differences in expression of trans-acting splicing factors necessary for correct splicing of the mutant allele. It seems reasonable to deduce that the predominantly ocular phenotype in this family is due to the ability to

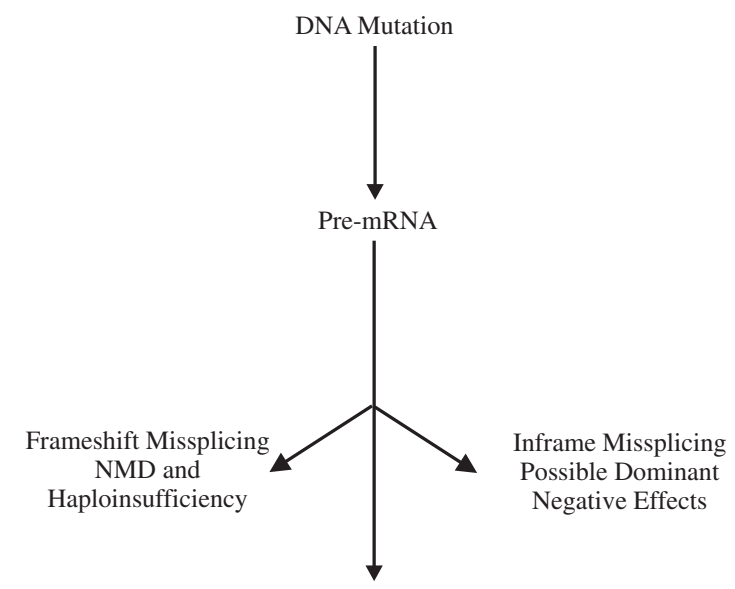

Normal Splicing

Figure 2 Possible effects of mutations on pre-mRNA processing. A mutation may result in either normal splicing or frameshifts that will often lead to incorporation of a premature termination codon into the new reading frame, leading to haploinsufficiency. Alternatively, inframe missplicing can result in mutant proteins that can exert a dominant-negative effect.

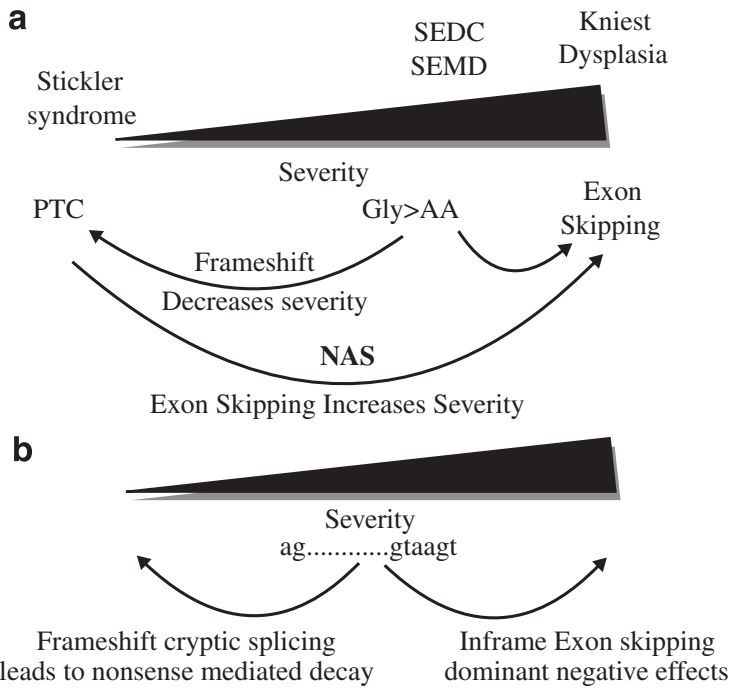

Figure 3 Missplicing can modify the phenotype of the type II collagenopathies. (a) Apparently similar mutations such as PTCs may be modified by nonsense-associated altered splicing (NAS). Even a small percentage of exon skipping resulting from PTCs will exert a dominant negative effect and modify the resulting phenotype, making it more severe. Similarly, missense mutations that interfere with pre-mRNA splicing can be modified either by exon skipping or by causing frameshifts. (b) Mutations of splice sites may result in the use of cryptic splice sites that result in NMD or exon skipping usually with dominant negative effects. The phenotype may vary depending on the proportion of each type of transcript.

splice the mutant allele normally. However, it is not clear whether this is due to differences in splicing efficiency between cartilage and ocular tissue or whether the 

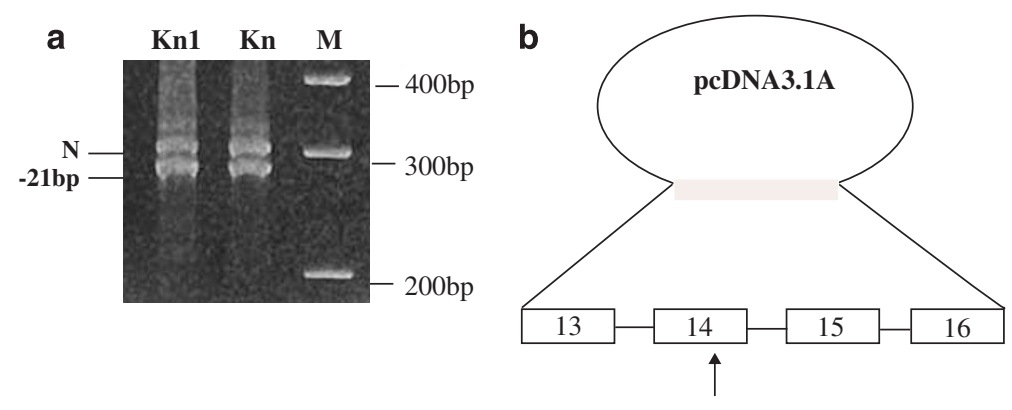

A102V

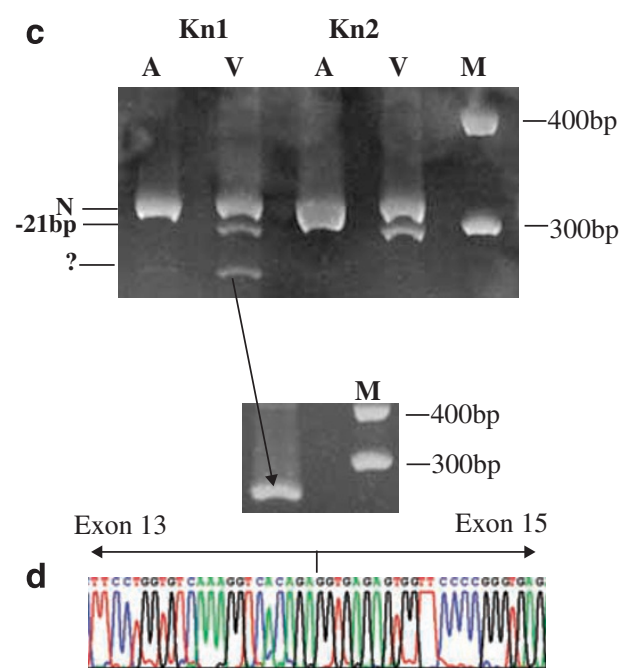

Figure 4 Variable splice products resulting from a mutant minigene. RT-PCR using illegitimate transcripts (a) from two cases of Kniest dysplaisa (Kn1 and Kn2) demonstrated utilisation of a de novo donor splice site and deletion of $21 \mathrm{bp}$ from the amplified cDNA. The A102V mutation cloned as minigenes (b) was transfected into the same patients' cell lines. Minigene-specific RT-PCR (c) amplified an additional product in Kn1 cells. Purification and sequencing (d) showed that this was due to skipping of exon 14.

development of the vitreous is more sensitive to reductions in the synthesis of type II collagen.

Other disease-causing mutations can also have a number of effects, not only by altering the amino-acid sequence but also by disrupting or creating splicing regulatory sequences (splicing enhancers or silencers) within the pre-mRNA. The effect of these mutations may vary from tissue to tissue and so apparently similar mutations, such as premature termination codons, can have dramatically different effects depending on whether they also affect pre-mRNA splicing and the degree, and tissue specificity of any missplicing that occurs and whether the transcript remains inframe or not (Figure 2). This nonsense-associated altered splicing (NAS) has been seen in a number of disorders. ${ }^{36,37}$ For collagen encoding genes, NAS resulting in exon skipping can have dramatic effects on phenotype, switching mutations that would normally result in haploinsufficiency, via nonsense mediated decay, into ones that have a dominant negative effect. This is because most exons in collagen genes encode complete
Gly-Xaa-Yaa amino-acid repeats and so exon skipping leaves the message inframe. Many missense (typically substitutions of obligate glycines within the collagen helix) and exon-skipping mutations in collagen genes have dominant negative effects because the mutant protein co-assembles with protein encoded by the normal allele to form abnormal trimeric collagen molecules. These structurally abnormal collagens are recognised by an endoplasmic reticulum-mediated quality control system, ${ }^{38}$ retained intracellularly and degraded. For a homotrimer, such as type II collagen, this means only $1 / 8$ of the molecule will be entirely normal with the remaining $7 / 8$ containing 1,2 , or 3 mutant $\alpha$-II collagen chains. These dominant-negative mutations typically result in severe phenotypes presenting clinically as spondyloepiphyseal dysplasia congenita (SEDC), spondyloepimetaphyseal dysplasia (SEMD), and Kniest dysplasia (MIM no. 183990, 184250, and 156550). Thus, the clinical outcome resulting from apparent nonsense and missense mutations can be modified by only a small difference in abnormal splicing (Figure 3). This 
Table 1 A selection of splicing mutations resulting in either Kniest dysplasia or Stickler's syndrome

\begin{tabular}{|c|c|c|}
\hline Mutation & Effect & Phenotype \\
\hline IVS9 c. $655-1 \mathrm{~g}>\mathrm{c}$ ASS tag $>$ tac & ND & Kniest/STL1 \\
\hline IVS16 c. $1023+1 \mathrm{~g}>\mathrm{t}$ DSS gt $>\mathrm{tt}$ & ND & Kniest \\
\hline IVS25 c. $1681-1 \mathrm{~g}>\mathrm{c}$ ASS aag $>$ aac & ND & STL1 \\
\hline IVS27 c. $1833+1 \mathrm{~g}>$ a DSS gt $>$ at & Cryptic splice-site utilisation & STL1 \\
\hline IVS28 c1887 $+1 \mathrm{~g}>\mathrm{a}$ DSS gt $>$ at & ND & STL1 \\
\hline IVS28 c1888-1g >c ASS ag > ac & ND & STL1 \\
\hline IVS29 c. $1941+1 \mathrm{~g}>$ a DSS gt $>$ at & ND & STL1 \\
\hline IVS39 c.2626-2a > g ASS ag > gg & ND & STL1 \\
\hline IVS51 c. $3886+1 \mathrm{~g}>\mathrm{t}$ DSS gt $>\mathrm{tt}$ & Exon skipping (frameshift + PTC) & STL1 \\
\hline IVS51 c3886 + 1g >a DSS gt $>$ at & Intron retention (PTC) & STL1 \\
\hline IVS51 c3886 + 2t $>$ c DSS gt $>$ gc & Exon skipping/normal splicing & POSTL1 \\
\hline
\end{tabular}

ASS, acceptor splice site; DSS, donor splice site; IVS, intervening sequence (intron); ND, not determined; POSTL1, predominantly ocular Stickler's syndrome; RT-PCR, reverse transcription PCR; STL1, Stickler's syndrome type 1.

Introns are numbered 1-53. Mutations are numbered in relation to the reference cDNA sequence NM_001844.3. This sequence has $157 \mathrm{bp}$ of a $5^{\prime}$ untranslated region. +1 corresponds to the A of the ATG translation initiation codon. Where performed, the effect of splicing has been determined by RT-PCR using illegitimate transcripts from cultured skin fibroblasts.

Table 2 Missense mutations in COL2A1 associated with mild phenotypes

\begin{tabular}{|c|c|c|}
\hline Mutation & Amino-acid change & Reference \\
\hline Exon 2 c. $170 \mathrm{~g}>\mathrm{a}$ & p.C57Y (N-propeptide) POSTL1 & 41 \\
\hline Exon 7 c. $431 \mathrm{~g}>\mathrm{t}^{\mathrm{a}}$ & p.G144V (N-propeptide) STL1 & 42 \\
\hline Exon 12 c. $801 \mathrm{~g}>\mathrm{a}$ & p.G267D (G67D) STL1 ${ }^{b}$ & 43 \\
\hline Exon 12 C.808g >c & p.G270R (G70R) STL1 & This laboratory (unpublished) \\
\hline Exon 13 c. $823 c>t$ & p.R275C (R75C) SP + Br & 44 \\
\hline Exon 15 c. $952 g>a^{a}$ & p.G318R (G118R) DRRD & 45 \\
\hline Exon $26 c .1693 c>t^{a}$ & p.R565C (R365C) STL1 & 19,44 \\
\hline Exon $31 c .1999 c>t^{a}$ & p.L667F (L467F) STL1/DRRD & 19 \\
\hline Exon 32 c. $2155 c>t$ & p.R719C (R519C) SP & 44 \\
\hline Exon 41 c. $2710 c>t$ & p.R904C (R704C) STL1 + Br & ${ }^{44,46}$ and this laboratory (unpublished) \\
\hline
\end{tabular}

Br, brachydactyly; DRRD, dominantly inherited rhegmatogenous retinal detachment; POSTL1, predominantly ocular Stickler's syndrome type 1; RTPCR, reverse transcription PCR; SP, spondyloarthropathy; STL1, Stickler's syndrome type 1.

Exons are numbered 1-54. Mutations are numbered in relation to the reference cDNA sequence NM_001844.3. This sequence has $157 \mathrm{bp}$ of a $5^{\prime}$ untranslated region. +1 corresponds to the A of the ATG translation initiation codon. Amino acids are numbered corresponding to the reference sequence NP_001835.2. Amino-acid substitutions within the collagen helix are also numbered (in parenthesis) according to the historical system whereby the first glycine of the collagen helix is 1 .

${ }^{\mathrm{a}}$ Mutations analysed by RT-PCR and/or as minigene splicing reporters in our laboratory.

${ }^{\mathrm{b}}$ The pG267D mutation was originally described as causing Wagner's vitreoretinal degeneration.

mechanism can also explain why the phenotype can vary between individuals with the same mutation depending on differences in the amount of missplicing that occurs between individuals.

We have studied two individuals with Kniest dysplasia and identical mutations, but subtly different vitreous phenotypes. One had disorganised vitreous gel architecture, whereas the other had the membranous vitreous phenotype commonly seen in patients with type II collagenopathies. The mutation has been seen previously in a number of cases of Kniest dysplasia. ${ }^{39,40}$ Despite altering the amino acid alanine 102 to valine, the pathogenic mechanism is missplicing, because it creates a de novo donor splice site in exon 14. Using cultured skin fibroblasts to examine illegitimate transcripts, we detected the same $21 \mathrm{bp}$ deletion from amplified cDNA that had been detected by others. However, when we transfected minigenes of the mutant allele back into the patients' own cells, exon skipping was detected, but only in one of the patient's cell lines (Figure 4). This variation is presumably due to a difference in splicing factors between the two cell lines. Whether this explains different vitreous phenotype is impossible to determine, but the two misspliced RNAs can potentially have different effects. Although the $21 \mathrm{bp}$ deletion leaves the message inframe, it disrupts the repetitive Gly-Xaa-Yaa protein sequence and so even mutant homotrimers will be recognised as abnormal and retained in the endoplasmic reticulum. In contrast, the 54 bp exon skip leaves the repetitive collagen sequence intact and mutant 

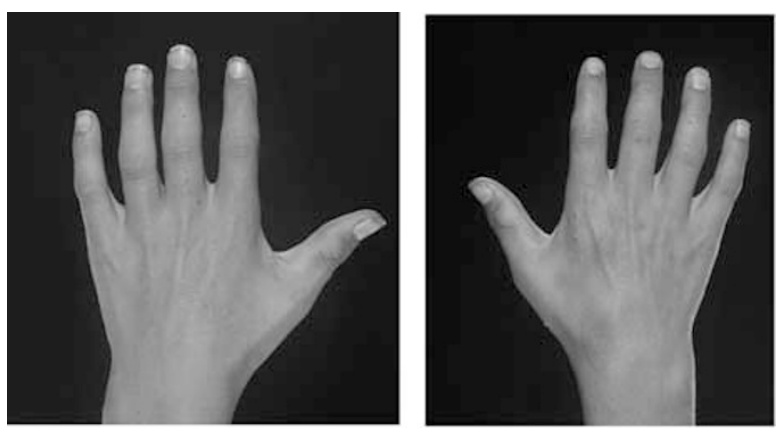

Arg904Cys
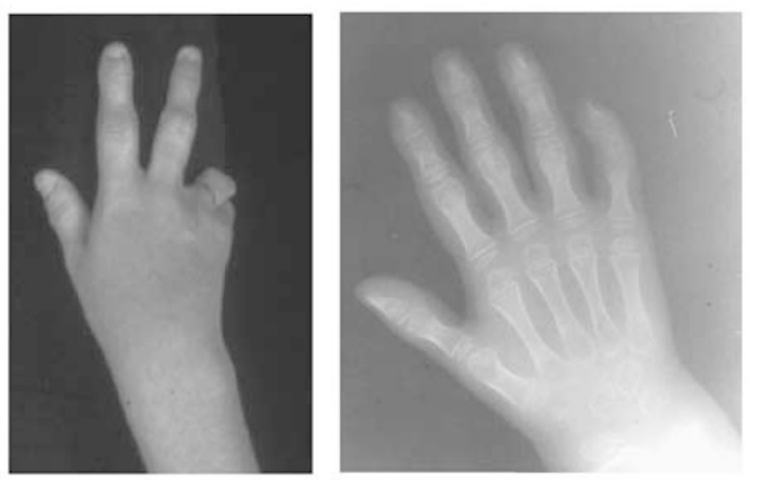

Kniest Dysplasia

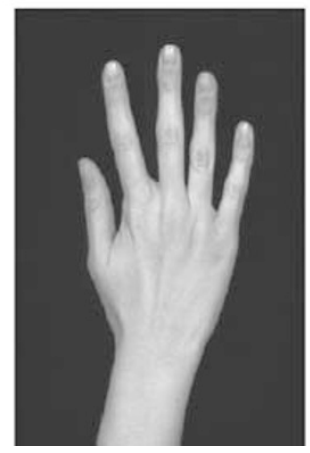

Arg565Cys

Figure 5 Variable brachydactyly seen in cases of arginine to cysteine mutations. A R904C mutation shows brachydactyly, as does an example of Kniest dysplasia. However, a R565C mutation does not.

homotrimers, although shorter than normal, can form a collagen triple helix and may be more efficiently secreted into the extracellular matrix.

Mutations of splice sites occur frequently in Stickler's syndrome; ${ }^{35}$ however, unlike those that cause exon skipping and result in Kniest dysplasia, the Stickler mutations appear to result in the utilisation of cryptic splice sites causing frameshifts and nonsense-mediated decay (Table 1). Our analysis of these mutations usually examines illegitimate transcripts from skin, but like the two cases of Kniest dysplasia discussed above, other splice products, such as exon skipping, may also result from these splice site mutations but go undetected in the illegitimate transcripts used for analysis. Hence, some of the phenotypic variability in these patients may be due to differences in the proportion of transcripts that are either targeted by the nonsense-mediated decay pathway (cryptic splice sites and frameshifts) or produce dominant negative effects (exon skips). These variations in mRNA splicing may differ both between cell types and also between individuals, and so modify the resulting phenotype (Figure 3b).

\section{Missense mutations}

Although most mutations causing type 1 Stickler's syndrome result in haploinsufficiency, there have been a few examples of missense mutations causing mild Stickler or Stickler-like phenotypes (Table 2). As discussed above, these mutations may have effects other than altering the amino-acid sequence, but it has also been shown that certain substitutions of glycine have less effect on the collagen molecule than others. ${ }^{47}$ Substitution of two consecutive glycines at positions 267 and 270 both result in mild phenotypes, suggesting that this region of the molecule is less sensitive to disruptions of the collagen helix. Others such as Arg904Cys produce additional phenotypic features, such as brachydactyly ${ }^{44}$ (Figure 5). This brachydactyly is more reminiscent of Kniest dysplasia and so may indicate that this mutation has additional effects on RNA processing than just the amino-acid change, such as exon skipping that commonly results in Kniest dysplasia. We have analysed some of these mutations (Table 2) either as illegitimate transcripts or as minigene splicing reporters and have not detected any missplicing, suggesting that their effect is through changes to the biophysical characteristics of the collagen. However, the Ala102Val and a silent mutation, ${ }^{42}$ that also creates a de novo donor splice site, demonstrate how single-base substitutions, including those thought to be missense mutations, may also have other pathogenic mechanisms that can modify the resulting phenotype. Recently, a missense mutation in exon 2 that converted Cys57Tyr has been described. ${ }^{41}$ The amino-acid substitution may be pathogenic, as it would be expected to alter the structure of the cysteinerich domain in the N-propeptide; however, the authors also demonstrated that it inhibits inclusion of exon 2 in the mature transcript. Although not expressed in mature cartilage, exon 2 is expressed in prechondrogenic nonocular tissues early in development ${ }^{48}$ and this may explain why some individuals with mutations in exon 2 show some systemic features of the disorder, such as cleft palate. ${ }^{28}$ It also indicates the importance of the 
cysteine-rich $\mathrm{N}$-propeptide domain in the normal development of the vitreous.

\section{Conclusion}

In the early to mid-1990s, the identification of mutations in the collagenopathies especially those of types I, II, and III collagens lead to attempts to correlate mutation (amino-acid change) and clinical phenotype. More recent studies have demonstrated how apparently similar and even identical mutations can have different effects on the processing of pre-mRNA. Along with the identification of common genetic variation that can predispose individuals to disorders, such as osteoporosis, osteoarthritis, and myopia, the concept of a simple genotype/phenotype correlation, that is, amino-acid change and clinical outcome would now appear to be somewhat outdated.

\section{Acknowledgements}

AJR was supported by Action Medical Research and Addenbrooke's NHS Trust. We thank Maureen Laidlaw for technical assistance. This study was supported by grants from Action Medical Research, The Evelyn Trust, and Addenbrooke's NHS Trust.

\section{References}

1 Stickler GB, Belau PG, Farrell FJ, Jones JD, Pugh DG, Steinberg AG et al. Hereditary progressive arthroophthalmopathy. Mayo Clin Proc 1965; 40: 433-455.

2 Snead MP, Yates JR. Clinical and molecular genetics of Stickler syndrome. J Med Genet 1999; 36: 353-359.

3 Blaschke UK, Eikenberry EF, Hulmes DJ, Galla HJ, Bruckner P. Collagen XI nucleates self-assembly and limits lateral growth of cartilage fibrils. J Biol Chem 2000; 275: 10370-10378.

4 Olsen BR. Collagen IX. Int J Biochem Cell Biol 1997; 29: 555-558.

5 Holden P, Meadows RS, Chapman KL, Grant ME, Kadler $\mathrm{KE}$, Briggs MD. Cartilage oligomeric matrix protein interacts with type IX collagen, and disruptions to these interactions identify a pathogenetic mechanism in a bone dysplasia family. J Biol Chem 2001; 276: 6046-6055.

6 Pihlajamaa T, Lankinen H, Ylostalo J, Valmu L, Jaalinoja J, Zaucke $\mathrm{F}$ et al. Characterization of recombinant aminoterminal NC4 domain of human collagen IX. J Biol Chem 2004; 279: 24265-24273.

7 Bishop PN, Holmes DF, Kadler KE, McLeod D, Bos KJ. Agerelated changes on the surface of vitreous collagen fibrils. Invest Ophthalmol Vis Sci 2004; 45: 1041-1046.

8 Ahmad NN, Ala-Kokko L, Knowlton RG, Jimenez SA, Weaver EJ, Maguire JI et al. Stop codon in the procollagen II gene (COL2A1) in a familiy with the Stickler syndrome (arthro-ophthalmopathy). Proc Natl Acad Sci USA 1991; 88: 6624-6627.
9 Maquat LE. When cells stop making sense: effects of nonsense codons on RNA metabolism in vertebrate cells. RNA 1995; 1: 453-465.

10 Tiller GE, Rimoin DL, Murray LW, Cohn DH. Tandem duplication within a type II collagen gene (COL2A1) exon in an individual with spondyloepiphyseal dysplasia. Proc Nat Acad Sci USA 1990; 87: 3889-3893.

11 Winterpacht A, Hilbert M, Schwarze U, Mundlos S, Spranger J, Zabel RU. Kniest and Stickler dysplasia phenotypes caused by collagen type II gene (COL2A1) defect. Nature Genet 1993; 3: 323-326.

12 Richards AJ, Yates JRW, Williams R, Payne SJ, Pope FM, Scott JD et al. A family with Stickler syndrome type 2 has a mutation in the COL11A1 gene resulting in the substitution of glycine 97 by valine in $\alpha 1(\mathrm{XI})$ collagen. Hum Mol Genet 1996; 5: 1339-1343.

13 Vikkula M, Mariman ECM, Lui VCH, Zhidkova NI, Tiller $\mathrm{GE}$, Goldring $\mathrm{MB}$ et al. Autosomal dominant and recessive osteochondrodysplasias associated with the COL11A2 locus. Cell 1995; 80: 431-437.

14 Van Camp G, Snoeckx RL, Hilgert N, van den Ende J, Fukuoka $\mathrm{H}$, Wagatsuma $\mathrm{M}$ et al. A new autosomal recessive form of Stickler syndrome is caused by a mutation in the COL9A1 gene. Am J Hum Genet 2006; 79: 449-457.

15 Muragaki Y, Mariman ECM, van Beersum SEC, Perala M, van Mourik JBA, Warman ML et al. A mutation in the gene encoding the alpha-2 chain of the fibril-associated collagen IX, COL9A2, causes multiple epiphyseal dysplasia (EDM2). Nature Genet 1996; 12: 103-105.

16 Paassilta P, Lohiniva J, Annunen S, Bonaventure J, Le Merrer M, Pai L et al. COL9A3: a third locus for multiple epiphyseal dysplasia. Am J Hum Genet 1999; 64: 1036-1044.

17 Czarny-Ratajczak M, Lohiniva J, Rogala P, Kozlowski K, Perala M, Carter L et al. A mutation in COL9A1 causes multiple epiphyseal dysplasia: further evidence for locus heterogeneity. Am J Hum Genet 2001; 69: 969-980.

18 Scott JD. Prevention and perspective in retinal detachment. Duke-Elder Lecture. Eye 1989; 3: 491-515.

19 Richards AJ, Baguley DM, Yates JR, Lane C, Nicol M, Harper PS et al. Variation in the vitreous phenotype of Stickler syndrome can be caused by different amino acid substitutions in the $X$ position of the type II collagen Gly-X-Y triple helix. Am J Hum Genet 2000; 67: 1083-1094.

20 Kizawa H, Kou I, Iida A, Sudo A, Miyamoto Y, Fukuda A et al. An aspartic acid repeat polymorphism in asporin inhibits chondrogenesis and increases susceptibility to osteoarthritis. Nat Genet 2005; 37: 138-144.

21 Miyamoto M, Mabuchi A, Shi D, Kubo T, Takatori Y, Saito S et al. A functional polymorphism in the $5^{\prime}$ UTR of GDF5 is associated with susceptibility to osteoarthritis. Nat Genet 2007; 39: 529-533.

22 Inamori $\mathrm{Y}$, Ota $\mathrm{M}$, Inoko $\mathrm{H}$, Okada E, Nishizaki R, Shiota $\mathrm{T}$ et al. The COL1A1 gene and high myopia susceptibility in Japanese. Hum Genet 2007; 122: 151-157.

23 Mutti DO, Cooper ME, O'Brien S, Jones LA, Maraita ML, Murray JC et al. Candidate gene and locus analysis of myopia. Mol Vis 2007; 13: 1012-1019.

24 Ikeda T, Mabuchi A, Fukuda A, Kawakami A, Yamada R, Yamamoto $\mathrm{S}$ et al. Association analysis of single nucleotide polymorphisms in cartilage-specific collagen genes with knee and hip osteoarthritis in the Japanese population. J Bone Miner Res 2002; 17: 1290-1296.

25 Noponen-Hietala N, Kylollen T, Mannikko M, Ikko E, Karppinen J, Ott J et al. Sequence variations in the collagen 
IX and XI genes are associated with degenerative lumbar spinal stenosis. Ann Rheum Dis 2003; 62: 1208-1214.

26 Sandell LJ, Morris N, Robbins JR, Goldring MB. Alternatively spliced type II procollagen mRNAs define distinct populations of cells during vertebral development: differential expression of the amino-propeptide. J Cell Biol 1991; 114: 1307-1319.

27 Zhidkova NI, Justice SK, Mayne R. Alternative mRNA processing occurs in the variable region of the pro- $\alpha 1(\mathrm{XI})$ and pro- $\alpha 2$ (XI) collagen chains. J Biol Chem 1995; 270: 9486-9493.

28 Richards AJ, Martin S, Yates JRW, Scott JD, Baguley DM, Pope FM et al. COL2A1 exon 2 mutations: relevance to the Stickler and Wagner syndromes. Br J Ophthalmol 2000; 84: 364-371.

29 Donoso LA, Edwards AO, Frost AT, Ritter R, Ahmad NN, Vrabec $\mathrm{T}$ et al. Identification of a stop codon mutation in exon 2 of the collagen 2A1 gene in a large stickler syndrome family. Am J Ophthalmol 2002; 134: 720-727.

30 Parma ES, Korkko J, Hagler WS, Ala-Kokko L. Radial perivascular retinal degeneration: a key to the clinical diagnosis of an ocular variant of Stickler syndrome with minimal or no systemic manifestations. Am J Ophthalmol 2002; 134: 728-734.

31 Miyamoto T, Inoue H, Sakamoto $\mathrm{Y}$, Kudo E, Naito T, Mikawa $\mathrm{T}$ et al. Identification of a novel splice site mutation of the CSPG2 gene in a Japanese family with Wagner syndrome. Invest Ophthalmol Vis Sci 2005; 46: 2726-2735.

32 Mukhopadhyay A, Nikopoulos K, Maugeri A, de Brouwer $\mathrm{AP}$, van Nouhuys CE, Boon CJ et al. Erosive vitreoretinopathy and Wagner disease are caused by intronic mutations in CSPG2/versican that result in an imbalance of splice variants. Invest Ophthalmol Vis Sci 2006; 47: 3565-3572.

33 Kloeckener-Gruissem B, Bartholdi D, Abdou MT, Zimmermann DR, Berger W. Identification of the genetic defect in the original Wagner syndrome family. Mol Vis 2006; 12: 350-355.

34 Matlin AJ, Clark F, Smith CWJ. Understanding alternative splicing: towards a cellular code. Nat Rev Mol Cell Biol 2005; 6: 386-398.

35 Richards AJ, Laidlaw M, Whittaker J, Treacy B, Rai H, Bearcroft $\mathrm{P}$ et al. High efficiency of mutation detection in type 1 stickler syndrome using a two-stage approach: vitreoretinal assessment coupled with exon sequencing for screening COL2A1. Hum Mut 2006; 27: 696-704.

36 Dietz HC, Valle D, Francomano CA, Kendzior Jr RJ, Pyritz RE, Cutting GR. The skipping of constitutive exons in vivo induced by nonsense mutations. Science 1993; 259: 680-683.
37 Liu, H-X, Cartegni L, Zhang MQ, Krainer AR. A mechanism for exon skipping caused by nonsense or missense mutations in BRCA1 and other genes. Nature Genet 2001; 27: 55-58.

38 Lamande SR, Bateman JF. Procollagen folding and assembly: the role of endoplasmic reticulum enzymes and molecular chaperones. Semin Cell Dev Biol 1999; 10: 455-464.

39 Bogaert R, Wilkin D, Wilcox WR, Lachman R, Rimoin D, Cohn DH et al. Expression, in cartilage, of a 7-amino-acid deletion in type II collagen from two unrelated individuals with Kniest dysplasia. Am J Hum Genet 1994; 55: 1128-1136.

40 Chen L, Yang W, Cole WG. Alternative splicing of exon 12 of the COL2A1 gene interrupts the triple helix of type-II collagen in the Kniest form of spondyloepiphyseal dysplasia. J Orthop Res 1996; 14: 712-721.

41 McAlinden A, Majave M, Bishop PN, Perveen R, Black GC, Pierpont ME et al. Missense and nonsense mutations in the alternatively-spliced exon 2 of COL2A1 cause the ocular variant of Stickler syndrome. Hum Mut 2008; 29: 83-90.

42 Richards AJ, Laidlaw ME, Meredith SP, Shankar P, Poulson AV, Scott JD et al. Missense and silent mutations in COL2A1 result in Stickler syndrome but via different molecular mechanisms. Hum Mut 2007; 28: 639.

43 Korkko J, Ritvaniemi P, Haataja L, Kaariainen H, Kivirikko KI, Prockop DJ et al. Mutation in type II procollagen (COL2A1) that substitutes aspartate for glycine alpha 1-67 and that causes cataracts and retinal detachment: evidence for molecular heterogeneity in the Wagner syndrome and the Stickler syndrome (arthroophthalmopathy). Am J Hum Genet 1993; 53: 55-61.

44 Hoornaert KP, Dewinter C, Vereecke I, Beemer FA, Courtens $\mathrm{W}$, Fryer A et al. The phenotypic spectrum in patients with arginine to cysteine mutations in the COL2A1 gene. J Med Genet 2006; 43: 406-413.

45 Richards AJ, Meredith S, Poulson A, Bearcroft P, Crossland $\mathrm{G}$, Baguley DM et al. A novel mutation of COL2A1 resulting in dominantly inherited rhegmatogenous retinal detachment. Invest Ophthalmol Vis Sci 2005; 46: 663-668.

46 Ballo R, Beighton PH, Ramesar RS. Stickler-like syndrome due to a dominant negative mutation in the COL2A1 gene. Am J Med Genet 1998; 80: 6-11.

47 Beck K, Chan VC, Shenoy N, Kirkpatrick A, Ramshaw JAM, Brodsky B. Destabilization of osteogenesis imperfecta collagen-like model peptides correlates with the identity of the residue replacing glycine. Proc Natl Acad Sci 1999; 97: 4273-4278.

48 Sandell LJ, Nalin AM, Reife RA. Alternative splice form of type II procollagen mRNA (IIA) is predominant in skeletal precursors and non-cartilaginous tissues during early mouse development. Dev Dyn 1994; 199: 129-140. 